\title{
Measurement of the acceptance by means of transverse beam excitation with noise
}

\author{
S. Sorge, ${ }^{*}$ G. Franchetti, and A. Parfenova \\ GSI Darmstadt, Planckstraße 1, 64291 Darmstadt, Germany
}

(Received 16 September 2009; published 31 May 2011)

\begin{abstract}
In this work, we present a technique to measure the acceptance of a circular accelerator extending a previous method by the measurement of particle loss. Here, we apply an rf voltage to transversely excite a coasting heavy ion beam in the SIS-18 synchrotron at GSI. The resulting growth of the transverse beam width causes particle loss when the beam width exceeds the limiting aperture. The acceptance is determined from the measured time evolution of the beam current after particles start to hit the aperture. In doing so, the result will not depend on the initial width of the ion beam.
\end{abstract}

DOI: 10.1103/PhysRevSTAB.14.052802

PACS numbers: $02.70 . N s, 29.20 . d k, 29.27 .-\mathrm{a}$

\section{INTRODUCTION}

The heavy ion synchrotron SIS-18 at GSI [1,2] is used for providing a large variety of heavy ions. Beam energies up to about $1 \mathrm{GeV} / \mathrm{u}$ for $\mathrm{U}^{73+}$ ions and $4.5 \mathrm{GeV}$ for protons are achievable. The variety of requirements concerning the operation, in particular, the usage of SIS-18 as a booster within the FAIR project [2,3] underway at GSI, also includes regular operation in a high-current regime. In this regime, uncontrolled beam loss in the pipe can lead to irradiation of the machine and vacuum degradation with a sensible reduction of the beam lifetime [4,5]. A precise knowledge of the SIS-18 acceptance will help to optimize the linear optics, which is the first step towards an improved beam loss control.

Limitations of the acceptance are caused by the accelerator optics of the injection scheme [6]. The integrated sum of all errors from the linear optics causes a reduction of the acceptance. Although the acceptance can theoretically be computed via particle tracking simulations, the lack of knowledge of an accelerator under real working conditions makes it desirable to develop a method for measuring the acceptance with beam.

Several experiments, which used the diffusion process, had been performed at different accelerators to measure the acceptance or the dynamic aperture. The particle diffusion was obtained by either using noise applied transversely to the beam [7] or using the ripple in the field of the magnets [8]. The latter method led to a stochastic detuning of the particles which generates transverse diffusion. The acceptance was determined from the measured time required for the beam edge to reach the aperture. Unfortunately, the estimation of this time had a large uncertainty because a detailed knowledge of the initial beam size was necessary

\footnotetext{
*S.Sorge@gsi.de
}

Published by the American Physical Society under the terms of the Creative Commons Attribution 3.0 License. Further distribution of this work must maintain attribution to the author(s) and the published article's title, journal citation, and DOI. which translated into a large uncertainty in the derived acceptance.

In order to avoid this uncertainty we study how the loss of particles evolves after they reach the limiting aperture of the accelerator. This means that the beam loss evolution is determined by stochastic dynamics rather than by the initial particle distribution. It can be shown that the integrated effect of the stochastic or pseudostochastic transverse $\mathrm{rf}$ voltage transforms any arbitrary initial particle distribution into the same asymptotic distribution allowing the determination of the accelerator acceptance.

This paper is organized into the following: Sec. II contains the derivation of an analytical model describing the growth of the beam size as a diffusion process driven by white noise. In Sec. III, the emittance growth and particle loss generated by a realistic rf field are discussed. In Sec. IV, a tracking model is introduced and compared to the diffusion model. In Sec. V the experimental data are presented and compared with results from simulations. Finally, the SIS-18 acceptance is determined from the data. The paper is concluded with a summary in Sec. VI and with Appendix A on the beam emittance growth due to white noise and Appendix B on the analysis and estimation of the acceptance error due to uncertainties in the lattice and beam parameters.

\section{DIFFUSION MODEL}

\section{A. Particle loss}

The method to measure the acceptance is based on the measurement of the time evolution of the beam current which is affected by noise driven beam loss. The noise propagates particles from regions of high density to weakly populated regions as shown by the equation

$$
\vec{j}=-C \nabla f,
$$

where only the motion in vertical phase space is considered. In Eq. (1), $f$ is the particle distribution function in the vertical phase space plane and $\nabla$ is the vector differential operator acting on the vertical normalized phase space 
coordinates $\left(y, p_{y}\right)=\left(y, \beta_{y} y^{\prime}+\alpha_{y} y\right)$, where $\alpha_{y}, \beta_{y}$ are the Twiss parameters. $C$ is the diffusion constant. Inserting Eq. (1) into the continuity equation

$$
\frac{\partial f}{\partial t}=-\nabla \cdot \vec{j}
$$

yields the diffusion equation

$$
\frac{\partial f}{\partial t}=C \nabla^{2} f
$$

Equation (3) can be used to calculate the beam growth and the resulting particle loss. To show that, we follow chapter 7.2 in Ref. [9] and restrict the discussion to an initial particle distribution function dependent only on the Courant-Snyder invariant $\epsilon=\left(y^{2}+p_{y}^{2}\right) / \beta_{y}$. This invariant is equal to the emittance of a particle. Furthermore, it is shown in Ref. [9] that $C$ can be replaced with $\beta_{y}\left(d \epsilon_{\mathrm{av}} / d t\right) / 4$ to include processes which occur at several locations in the ring, where $\epsilon_{\mathrm{av}}$ is the emittance averaged over all particles in the beam. The diffusion equation in terms of emittances becomes

$$
\frac{\partial f}{\partial t}=\left(\frac{d \epsilon_{\mathrm{av}}}{d t}\right) \frac{\partial}{\partial \epsilon}\left(\epsilon \frac{\partial f}{\partial \epsilon}\right) .
$$

This equation has to be solved for the boundary conditions

$$
f\left(\epsilon=\epsilon_{\mathrm{lim}}, t\right)=0 \quad \text { and } \quad f(\epsilon, t=0)=f_{0}(\epsilon),
$$

where the first constraint means that a particle becomes lost when its emittance $\epsilon$ reaches the acceptance $\epsilon_{\text {lim }}$. The general solution to Eq. (4) is

$$
f(\epsilon, t)=\sum_{n=1}^{\infty} c_{n} J_{0}\left(\lambda_{n} \sqrt{\frac{\epsilon}{\epsilon_{\mathrm{lim}}}}\right) \exp \left[-\frac{\lambda_{n}^{2}}{4}\left(\frac{d \epsilon_{\mathrm{av}}}{d t}\right) \frac{t}{\epsilon_{\mathrm{lim}}}\right]
$$

with $0 \leq \epsilon \leq \epsilon_{\text {lim }}$. Here, $c_{n}$ are constant coefficients determined by $f_{0}(\epsilon), J_{0}$ is a Bessel function, and $\lambda_{n}$ are the zeros of $J_{0}$. Note that $f(\epsilon, t)$ is a solution to Eq. (4) only if the condition

$$
\frac{d \epsilon_{\mathrm{av}}}{d t}=\mathrm{const}
$$

is fulfilled. This can be proven by inserting the right-hand side of Eq. (6) into Eq. (4) and requiring that $J_{0}$ be a solution to Bessel's equation, $x^{2} J_{0}^{\prime \prime}+x J_{0}^{\prime}+x^{2} J_{0}=0$. It will be shown in the following section that $d \epsilon_{\mathrm{av}} / d t$ is a constant which is independent of the initial particle distribution.

The summands on the right-hand side of Eq. (6) decrease exponentially in time with the characteristic time for the decrease determined by the $\lambda_{n}$. $\lambda_{1}$ is the smallest zero of $J_{0}$. Therefore, the first summand decreases much slower than all other terms. When $t$ becomes sufficiently large so that the condition $\left(d \epsilon_{\mathrm{av}} / d t\right)\left(t / \epsilon_{\mathrm{lim}}\right) \sim 1+\ln \left|c_{n \text {, } \max } / c_{1}\right|$ is approximately fulfilled, $f(\epsilon, t)$ will essentially be given by its first summand. Here, $c_{n \text {, max }}$ denotes the coefficient with the largest modulus. In this case the distribution function approximately reads

$$
f(\epsilon, t) \propto J_{0}\left(\lambda_{1} \sqrt{\frac{\epsilon}{\epsilon_{\mathrm{lim}}}}\right) \exp \left[-\frac{\lambda_{1}^{2}}{4}\left(\frac{d \epsilon_{\mathrm{av}}}{d t}\right) \frac{t}{\epsilon_{\mathrm{lim}}}\right] .
$$

The exponential function causes only an exponential decrease of $f(\epsilon, t)$ but does not affect its spatial shape. Therefore, the spatial shape is given by the Bessel function $J_{0}\left(\lambda_{1} \sqrt{\epsilon / \epsilon_{\text {lim }}}\right)$ which has its maximum value at $\epsilon=0$ and is monotonically decreasing with increasing $\epsilon$ for $\epsilon \leq \epsilon_{\mathrm{lim}}$. Integrating Eq. (8) over $\epsilon$, one finds the time dependent number of particles

$$
N(t) \propto \exp \left[-\frac{\lambda_{1}^{2}}{4}\left(\frac{d \epsilon_{\mathrm{av}}}{d t}\right) \frac{t}{\epsilon_{\mathrm{lim}}}\right] .
$$

Equations (8) and (9) show that for large $t$ the time evolution of $f(\epsilon, t)$ and $N(t)$ do not depend on the initial particle distribution. However, in a real measurement a beam width smaller than the limiting aperture should be used, otherwise most of the particles will be lost before the memory of the initial beam shape can be washed out by the noise.

When Eq. (9) can be applied we find the expression

$$
\epsilon_{\lim }=\frac{\lambda_{1}^{2}}{4}\left(\frac{d \epsilon_{\mathrm{av}}}{d t}\right) \tau_{\mathrm{loss}}
$$

where

$$
\tau_{\text {loss }}=\left(t_{2}-t_{1}\right)\left[\ln \left(\frac{N\left(t_{1}\right)}{N\left(t_{2}\right)}\right)\right]^{-1}
$$

is the characteristic loss time and $t_{1}, t_{2}$ are arbitrary moments.

\section{B. Time dependent emittance}

The time dependent averaged emittance $\epsilon_{\mathrm{av}}$ written as a function of the revolution number $n$ is

$$
\epsilon_{\mathrm{av}}(n)=\frac{1}{N_{p}} \sum_{p=1}^{N_{p}} \epsilon_{p}(n)
$$

where

$$
\epsilon_{p}(n)=\gamma_{y} y_{p, n}^{2}+2 \alpha_{y} y_{p, n} y_{p, n}^{\prime}+\beta_{y} y_{p, n}^{\prime 2}
$$

is the emittance of the $p$ th particle and $\alpha_{y}, \beta_{y}, \gamma_{y}$ are the Twiss parameters at the location of the source of the noise. The evolution of the phase space coordinates of a single particle follows

$$
\left(\begin{array}{l}
y_{p, 1} \\
y_{p, 1}^{\prime}
\end{array}\right)=M\left(\begin{array}{c}
y_{p, 0} \\
y_{p, 0}^{\prime}+\Delta y_{p, 0}^{\prime}
\end{array}\right)=M\left(\begin{array}{c}
y_{p, 0} \\
y_{p, 0}^{\prime}
\end{array}\right)+M\left(\begin{array}{c}
0 \\
\Delta y_{p, 0}^{\prime}
\end{array}\right)
$$

where

$$
M=\left(\begin{array}{cc}
\cos \mu+\alpha_{y} \sin \mu & \beta_{y} \sin \mu \\
-\frac{1+\alpha_{y}^{2}}{\beta_{y}} \sin \mu & \cos \mu-\alpha_{y} \sin \mu
\end{array}\right)
$$

is the one turn map, and $\mu=2 \pi \nu_{y}$ is the phase advance of the betatron oscillation. $\Delta y_{p, 0}^{\prime}$ is the momentum kick due to 
the noise. The characteristics of the noise are introduced later.

Iterating Eq. (14) yields the phase space coordinates of a single particle after $n$ revolutions,

$$
\left(\begin{array}{l}
y_{p, n} \\
y_{p, n}^{\prime}
\end{array}\right)=M^{n}\left(\begin{array}{l}
y_{p, 0} \\
y_{p, 0}^{\prime}
\end{array}\right)+\sum_{k=0}^{n-1} M^{n-k}\left(\begin{array}{c}
0 \\
\Delta y_{p, k}^{\prime}
\end{array}\right) .
$$

Using this equation, Eq. (12), and Eq. (13), we obtain

$$
\begin{aligned}
\epsilon_{\mathrm{av}}(n)= & \frac{1}{N_{p}} \sum_{p=1}^{N_{p}}\left(y_{p, n}, y_{p, n}^{\prime}\right) \cdot B \cdot\left(\begin{array}{c}
y_{p, n} \\
y_{p, n}^{\prime}
\end{array}\right) \\
= & \frac{1}{N_{p}} \sum_{p=1}^{N_{p}}\left\{\left(y_{p, 0}, y_{p, 0}^{\prime}\right) \cdot\left(M^{n}\right)^{T} \cdot B \cdot M^{n} \cdot\left(\begin{array}{c}
y_{p, 0} \\
y_{p, 0}^{\prime}
\end{array}\right)\right. \\
& +\sum_{k=0}^{n-1}\left[\left(y_{p, 0}, y_{p, 0}^{\prime}\right) \cdot\left(M^{n}\right)^{T} \cdot B \cdot M^{n-k} \cdot\left(\begin{array}{c}
0 \\
\Delta y_{p, k}^{\prime}
\end{array}\right)\right. \\
& \left.+\left(0, \Delta y_{p, k}^{\prime}\right) \cdot\left(M^{n-k}\right)^{T} \cdot B \cdot M^{n}\left(\begin{array}{c}
y_{p, 0} \\
y_{p, 0}^{\prime}
\end{array}\right)\right] \\
& \left.+\sum_{k, l=0}^{n-1}\left(0, \Delta y_{p, l}^{\prime}\right) \cdot\left(M^{n-l}\right)^{T} \cdot B \cdot M^{n-k} \cdot\left(\begin{array}{c}
0 \\
\Delta y_{p, k}^{\prime}
\end{array}\right)\right\},
\end{aligned}
$$

where the upper index $T$ denotes the transpose of a matrix. $B$ is the beta matrix defined by

$$
B=\left(\begin{array}{cc}
\frac{1+\alpha_{y}^{2}}{\beta_{y}} & \alpha_{y} \\
\alpha_{y} & \beta_{y}
\end{array}\right) .
$$

The momentum kicks $\Delta y_{p, k}^{\prime}$ in Eq. (17) are assumed to be uncorrelated with respect to the particle number $p$ and the revolution number $k$. Hence, the kicks are entirely determined by a stationary single-kick distribution function $p_{1}\left(\Delta y^{\prime}\right)$. Under this assumption, the average of any function of the momentum kick, $g\left(\Delta y^{\prime}\right)$, can be approximated by

$$
\int_{-\infty}^{\infty} g\left(\Delta y^{\prime}\right) p_{1}\left(\Delta y^{\prime}\right) d\left(\Delta y^{\prime}\right)=\frac{1}{N} \sum_{n=1}^{N} g\left(\Delta y_{n}^{\prime}\right)
$$

if $N$ is sufficiently large. The mean value of the distribution function is assumed to be

$$
\int_{-\infty}^{\infty} \Delta y^{\prime} p_{1}\left(\Delta y^{\prime}\right) d\left(\Delta y^{\prime}\right)=0
$$

and we define the mean square value

$$
\int_{-\infty}^{\infty}\left(\Delta y^{\prime}\right)^{2} p_{1}\left(\Delta y^{\prime}\right) d\left(\Delta y^{\prime}\right)=\sigma_{\Delta y^{\prime}}^{2}
$$

Applying this in Eq. (17) leads to

$$
\epsilon_{\mathrm{av}}(n)=\epsilon_{\mathrm{av}, 0}+n \beta_{y} \sigma_{\Delta y^{\prime}}^{2} .
$$

The derivation of this formula is shown in Appendix A.

The time derivative of the averaged emittance given in Eq. (22) is

$$
\left.\frac{d \epsilon_{\mathrm{av}}(t)}{d t}\right|_{t=n T_{0}} \approx \frac{\epsilon_{\mathrm{av}}(n+1)-\epsilon_{\mathrm{av}}(n)}{T_{0}}=\frac{\beta_{y} \sigma_{\Delta y^{\prime}}^{2}}{T_{0}} .
$$

Here, $T_{0}$ is the revolution time. Using this result in Eq. (10), one finds

$$
\epsilon_{\lim }=\frac{\lambda_{1}^{2}}{4} \beta_{y} \sigma_{\Delta y^{\prime}}^{2} \frac{\tau_{\text {loss }}}{T_{0}}
$$

\section{BEAM BEHAVIOR AFFECTED BY RF EXCITATION}

\section{A. The rf exciter}

In the previous section we have discussed the growth of the beam width arising from particle diffusion driven by a purely stochastic force. In reality the force acting on the particles is, basically, a sinusoidal signal generated by an $\mathrm{rf}$ exciter. The basic parameters of the exciter as well as those of the beam and the lattice are presented in Table I. More details can be found in Ref. [10].

The exciter consists of two parallel plates with the distance $d_{0}$ between each other. Between them the voltage

$$
U(t)=U_{a} \sin \left[2 \pi f_{C} t+\phi_{0}(t)\right]
$$

is generated, where $f_{C}$ is the carrier or main frequency, and $U_{a}$ is the amplitude voltage. The sinusoidal signal is modified by the phase $\phi_{0}(t)$. The phase is determined by a pseudorandom bit sequence, where it is $\phi_{0}=\pi$ during a period when the bit status is 1 and $\phi_{0}=0$ otherwise. The duration of a bit status is $T_{S}=1 / f_{S}$, where $f_{S}$ is the bit rate of the pseudorandom generator.

The power spectrum of the rf voltage is

$$
P(f) \propto \frac{\sin ^{2}\left[\pi\left(f-f_{C}\right) / f_{S}\right]}{\left[\left(f-f_{C}\right) / f_{S}\right]^{2}}+\frac{\sin ^{2}\left[\pi\left(f+f_{C}\right) / f_{S}\right]}{\left[\left(f+f_{C}\right) / f_{S}\right]^{2}} .
$$

\section{TABLE I. Main experimental parameters.}

\begin{tabular}{lc}
\hline \hline Circumference of SIS-18C & $216.72 \mathrm{~m}$ \\
Working point $\nu_{x}, \nu_{y}$ & $4.17,3.29$ \\
Ion & $\mathrm{Ta}^{61+}$ \\
Initial ion number $N_{p}(0)$ & $\sim 10^{9}$ \\
Particle energy $E$ & $(100,200) \mathrm{MeV} / \mathrm{u}$ \\
Revolution time $T_{0}$ at $(100,200) \mathrm{MeV} / \mathrm{u}$ & $(1.683,1.273) \mu \mathrm{s}$ \\
Measuring time $t_{\text {max }}$ & $\approx 1.8 \mathrm{~s}$ \\
Resulting revolution number $n$ & $\sim 10^{6}$ \\
rms momentum width $\sigma_{p}$ & $5.0 \times 10^{-4}$ \\
Nominal vertical chromaticity $\xi_{y, \text { nat }}$ & -1.4647 \\
Exciter length $l_{0}$ & $750 \mathrm{~mm}$ \\
Vertical exciter plate distance $d_{0}$ & $70 \mathrm{~mm}$ \\
Exciter amplitude voltage $U_{a}$ & $14.5 \mathrm{~V}$ and $29 \mathrm{~V}$ \\
Exciter frequency width $f_{S}$ & $0.005 / T_{0}$ and $0.01 / T_{0}$ \\
$\beta_{y}$ at exciter position & $7.0 \mathrm{~m}$ \\
$\alpha_{y}$ at exciter position & 0.15 \\
\hline \hline
\end{tabular}


We omit the second term because it corresponds to negative frequencies. At $f=f_{C} \pm f_{S}$, one finds the zeros of $P(f)$ closest to the carrier frequency. So, $f_{S}$ is the half width at zero spectral power density of the central peak of $P(f)$.

The voltage $U(t)$ leads to a time dependent, transverse electric field with an amplitude field strength

$$
E_{a}=\frac{U_{a}}{d_{0}} .
$$

Because the length of the exciter is much smaller than the length of a betatron period, the exciter acts approximately as a thin lens. Hence, the electric field creates in each revolution a momentum kick to the particles with a sinusoidal time behavior:

$$
\Delta y_{n}^{\prime}=\Delta y_{a}^{\prime} \sin \left[n \psi_{C}+\phi_{0}(n)\right] .
$$

Its amplitude value is

$$
\Delta y_{a}^{\prime}=\frac{q E_{a} \Delta t}{p},
$$

where $q$ is the ion charge, $p$ is the momentum, and $\Delta t$ is the time needed by an ion to pass through the exciter. Using $q=Z e, \Delta t=l_{0} /\left(\beta_{0} c\right)$, and $p=A m_{u} c \beta_{0} \gamma_{0}$, this equation can be rewritten to

$$
\Delta y_{a}^{\prime}=\frac{e}{m_{u} c^{2} \beta_{0}^{2} \gamma_{0}} \frac{Z}{A} \frac{U_{a} l_{0}}{d_{0}} .
$$

Here, $m_{u} c^{2}=931.5 \mathrm{MeV}$ is the rest energy of a nucleon, $\beta_{0}, \gamma_{0}$ are the relativistic factors, $l_{0}=750 \mathrm{~mm}$ is the length of the exciter, $d_{0}=70 \mathrm{~mm}$ is the distance between the plates, and $Z, A$ are charge state and the mass number of the ions used.

In order to reach a maximum efficiency in beam excitation, the phase advance of the sinusoidal momentum kick is set to

$$
\psi_{C}=2 \pi f_{C} T_{0}=2 \pi \nu_{y, \text { frac }},
$$

because the excitation efficiency is approximately proportional to the spectral power density at $f=\nu_{y, \text { frac }} / T_{0}$.

The main difference with respect to the noise assumed in Sec. II is that the rf signal from the exciter generates the same momentum kick to all particles. Hence, the mean square value of the momentum kicks is

$$
\sigma_{\Delta y^{\prime}}^{2}=\lim _{N \rightarrow \infty} \frac{1}{N} \sum_{n=0}^{N}\left(\Delta y_{n}^{\prime}\right)^{2} .
$$

The consequences of the application of such a momentum kick are discussed in the following section.

\section{B. Emittance growth due to the rf signal}

The major consequence of using an rf signal creating a uniform kick to all particles is that all particles will move coherently and independently of the particle density in phase space. Consequently, particle diffusion will not happen. Instead, the entire beam will randomly oscillate. On the other hand, the momentum spread $\delta=\Delta p / p$ will lead
TABLE II. Conditions used in the test tracking calculations presented in this section.
Particle energy $E$

Exciter amplitude voltage $U_{a}$ rms momentum uncertainty $\sigma_{p}$ Chromaticity $\xi_{y}$

Carrier frequency $f_{C}=\nu_{y \text {, frac }} / T_{0}$

Particles used in the simulations
$100 \mathrm{MeV} / \mathrm{u}$

$29 \mathrm{~V}$

0 and $5.0 \times 10^{-4}$

$0,-0.01,-0.1,-1.0$

$172.3 \mathrm{kHz}$

$1000, \ldots, 5000$ to a spread in the particle tune, $\Delta \nu=\nu_{y} \xi_{y} \delta$, where $\xi_{y}$ is the normalized chromaticity. Particle tracking simulations were performed to investigate the influence of this tune spread. The details of the tracking algorithm are introduced later; the parameters are presented in Table II. The simulations show that the oscillation of the beam center vanishes due to Landau damping when the tune spread is sufficiently large. The resulting beam profile has the maximum at the center and is monotonically decreasing with increasing radius, which was pointed out in Sec. II to be a consequence of particle motion driven by diffusion after a sufficiently long time. Additionally, chromaticity has a smoothing influence on the growth of the beam emittance $\epsilon_{\mathrm{av}}(t)$, which one can see in Fig. 1. For $\left|\xi_{y}\right|>0.1$, the emittance no longer depends on the chromaticity and is approximately a linear function of time, which is in qualitative agreement with Eq. (23). The quantitative differences resulting from the different types of noise are discussed in the next section.

\section{MODEL FOR PARTICLE TRACKING}

The analytic model discussed in Sec. II is not applicable for the quantitative determination of the acceptance

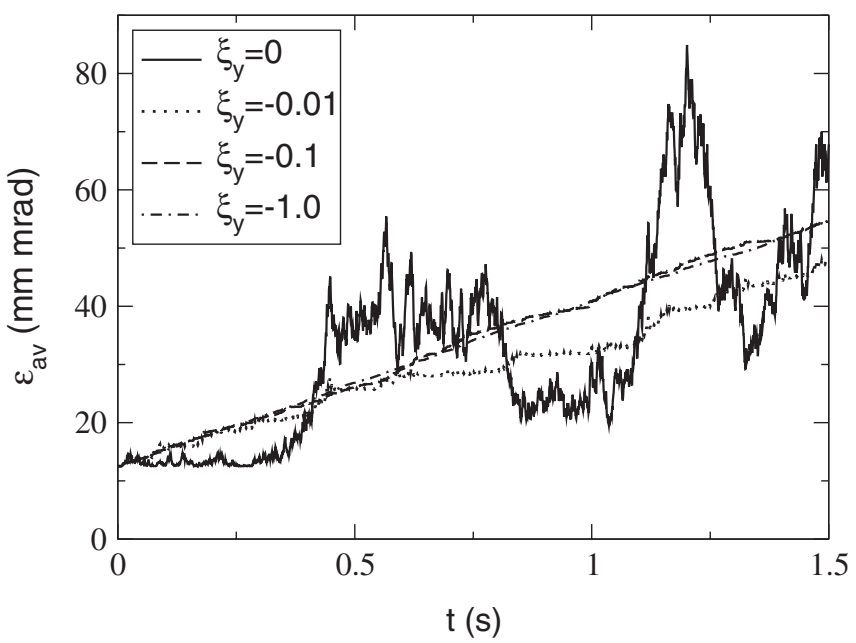

FIG. 1. Time evolution of the beam emittance calculated using the parameters from Table II depending on the chromaticity $\xi_{y}$. For $\left|\xi_{y}\right|>0.1$ the growth of the emittance does not depend on chromaticity. Consequently, the curves for $\xi_{y}=-0.1$ and $\xi_{y}=-1$ are approximately identical. 
because the real $\mathrm{rf}$ signal differs from white noise. Hence we study the problem numerically. The experiment is modeled by tracking particles through a lattice where a momentum kick identical to that of the rf exciter is applied to each particle for every turn. The time behavior of the beam emittance and the number of remaining particles are determined by assuming a test acceptance. The machine acceptance is obtained with a fitting procedure which varies the test acceptance until there is good agreement between the calculated and the measured particle number.

In our modeling we assume a linear lattice with decoupled horizontal and vertical particle motions. This enables us to use a one-dimensional model in our tracking calculations. We use a simplified lattice represented by the one turn map $M$ defined in Eq. (15), so that the particle motion is given by Eq. (14). The momentum kick $\Delta y_{n}^{\prime}$ has the sinusoidal time behavior given by Eq. (28). The initial particle coordinates are chosen according to a bi-Gaussian distribution in the vertical phase space and a Gaussian distribution in the longitudinal momentum space. That allows us to take into account the effect of the momentum spread in case of finite chromaticity. For this reason, the one turn map from Eq. (15) is modified to

$M=M\left(\mu_{p}\right)=\left(\begin{array}{cc}\cos \mu_{p}+\alpha_{y} \sin \mu_{p} & \beta_{y} \sin \mu_{p} \\ -\frac{1+\alpha_{y}^{2}}{\beta_{y}} \sin \mu_{p} & \cos \mu_{p}-\alpha_{y} \sin \mu_{p}\end{array}\right)$

with the modified phase advance,

$$
\mu_{p}=\mu+\mu \xi_{y} \delta_{p}
$$

where $\delta_{p}$ is the individual momentum deviation of the $p$ th particle. We assume $\delta_{p}=$ const because the beam is assumed to be coasting. $\alpha_{y}$ and $\beta_{y}$ are assumed to be not affected by $\delta_{p}$.

We benchmarked this model against the diffusion model presented in Sec. II. Here, we applied entirely stochastic momentum kicks to the particles to simulate diffusion due to white noise. In the comparison we found very good agreement between the tracking results and the analytic model. In particular, we found that the functional dependence between turn number $n=t / T_{0}$ and particle number $N(n)$ follows a scaling of $n \beta_{y} \sigma_{\Delta y^{\prime}}^{2} / \epsilon_{\text {lim }}$ which agrees with Eq. (9), where Eq. (23) is inserted.

\section{EXPERIMENTAL RESULTS AND THEIR EVALUATION}

\section{A. Model validation}

As the first step, we perform a benchmarking test on the applicability of the diffusion model introduced in Sec. II. We apply a local bump to the closed orbit, thus shifting the beam by a defined displacement towards the aperture of the ring. This causes an artificial reduction of the acceptance and so faster particle loss. According to Eq. (10) we expect

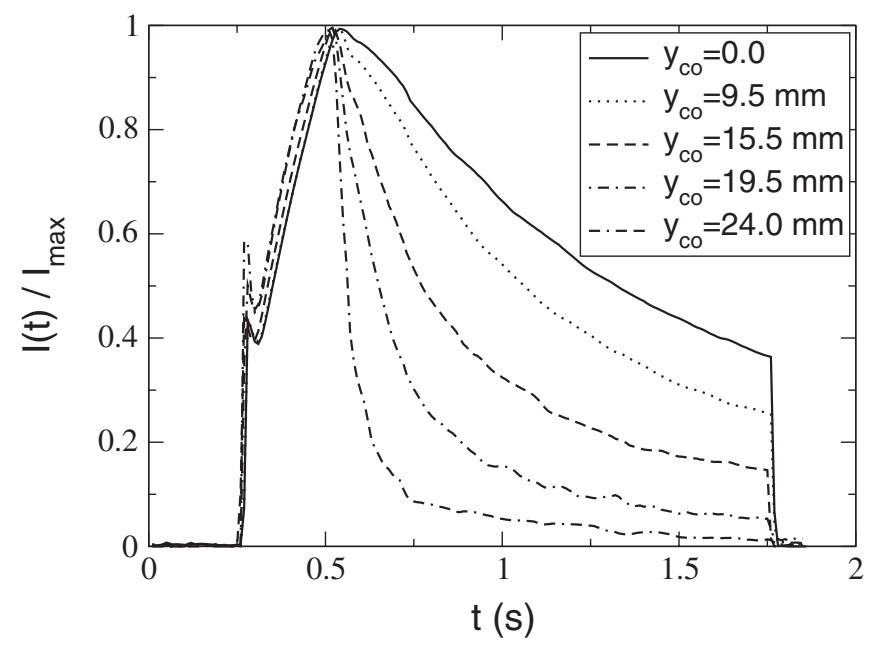

FIG. 2. Current as a function of time for different displacements due to the local closed orbit bump $y_{\mathrm{co}}$. The injection starts at $t=0.25 \mathrm{~s}$, the bump is applied from $t=0.5 \mathrm{~s}$, and the beam is extracted at about $t=1.8 \mathrm{~s}$. The exciter amplitude voltage $U_{a}=29 \mathrm{~V}$, the half width of the power spectrum of the exciter [Eq. (26)] $f_{S}=0.005 / T_{0}$, and the beam energy $E=100 \mathrm{MeV} / \mathrm{u}$ were chosen.

a characteristic loss time $\tau_{\text {loss }} \propto \epsilon_{\text {lim }} \propto y_{\text {lim }}^{2}$, where $y_{\text {lim }}$ is the limiting aperture. We present, here, results obtained for a beam energy $E=100 \mathrm{MeV} / \mathrm{u}$ and an exciter signal which has an amplitude voltage $U_{a}=29 \mathrm{~V}$ and a power spectrum with the half width $f_{S}=0.005 / T_{0}$ and the carrier frequency $f_{C}=\nu_{y, \text { frac }} / T_{0}$, where $\nu_{y \text {, frac }}=0.29$. Figure 2 shows the current measured under these conditions as a function of time. The displacement of the closed orbit, $y_{\mathrm{co}}$, measured at the location of a beam position monitor (BPM), $s=s_{\mathrm{BPM}}$, and the resulting loss times $\tau_{\text {loss }}$ defined by Eq. (11) and normalized to the revolution time $T_{0}$ are presented in Table III.

The previous section showed that, in spite of the difference between the realistic rf signal and white noise, the averaged emittance grows linearly in time which yields an exponential decrease of the particle number, if the chromaticity is

TABLE III. Normalized loss times, $\tau_{\text {loss }} / T_{0}$, for $U_{a}=29 \mathrm{~V}$, $f_{S}=0.005 / T_{0}$, and $E=100 \mathrm{MeV} / \mathrm{u}$ depending on the height of the closed orbit bump at the location of the beam position monitor, $s=s_{\mathrm{BPM}}$. The loss times were determined from the duration of the time interval when $0.7 I_{\max } \geq I(t) \geq 0.4 I_{\max }$, see Fig. 2. The vertical beta function at $s_{\mathrm{BPM}}$ is assumed to be $\beta_{y}=19.5 \mathrm{~m}$ according to a calculation using MAD-X.

\begin{tabular}{lcc}
\hline \hline Index $i$ & $y_{\mathrm{co}, i} / \mathrm{mm}$ & $\tau_{\text {loss }, i} / T_{0}$ \\
\hline 0 & 0 & $7.15 \times 10^{5}$ \\
1 & 9.5 & $4.67 \times 10^{5}$ \\
2 & 15.5 & $2.27 \times 10^{5}$ \\
3 & 19.5 & $1.11 \times 10^{5}$ \\
4 & 24.0 & $2.98 \times 10^{4}$ \\
\hline \hline
\end{tabular}


sufficiently large. This allows us to construct an analytical dependence between the displacement due to the closed orbit bump and the resulting loss times. We write Eq. (24) as

$$
\epsilon_{\lim }=\eta \frac{\tau_{\text {loss }}}{T_{0}},
$$

where $\eta$ will be found with a regression analysis using the loss times, $\tau_{\text {loss }, i}$, from Table III. If, furthermore, the closed orbit bump is large enough so that particle loss occurs basically only at its maximum position, $s=s_{\max }$, we can replace the left-hand side of Eq. (35) by the acceptance reduced by the closed orbit bump given at $s_{\max }$ :

$$
\epsilon_{\lim }\left(y_{\mathrm{co}}, s_{\max }\right)=\frac{\left[y_{\lim }\left(s_{\max }\right)-y_{\mathrm{co}}\left(s_{\max }\right)\right]^{2}}{\beta_{y}\left(s_{\max }\right)},
$$

where $y_{\lim }\left(s_{\max }\right)$ is the limiting aperture at $s_{\max }$. Expanding this expression and defining

$$
\epsilon_{\lim , 0}\left(s_{\max }\right)=\frac{y_{\lim }^{2}\left(s_{\max }\right)}{\beta_{y}\left(s_{\max }\right)}
$$

yields

$$
\begin{aligned}
\epsilon_{\lim }\left(y_{\mathrm{co}}, s_{\max }\right)= & \epsilon_{\lim , 0}\left(s_{\max }\right)-2 \frac{y_{\lim }\left(s_{\max }\right)}{\beta_{y}\left(s_{\max }\right)} y_{\mathrm{co}}\left(s_{\max }\right) \\
& +\frac{y_{\mathrm{co}}^{2}\left(s_{\max }\right)}{\beta_{y}\left(s_{\max }\right)} .
\end{aligned}
$$

Assuming the shape of the closed orbit bump is independent of its height it is

$$
y_{\mathrm{co}}\left(s_{\max }\right)=A y_{\mathrm{co}}\left(s_{\mathrm{BPM}}\right),
$$

where $A$ is a constant. Using that in Eq. (38), and inserting Eq. (38) into Eq. (35), we find

$$
c_{0}+c_{1} y_{\mathrm{co}}\left(s_{\mathrm{BPM}}\right)+c_{2} y_{\mathrm{co}}^{2}\left(s_{\mathrm{BPM}}\right)=\frac{\tau_{\mathrm{loss}}\left(y_{\mathrm{co}}\right)}{T_{0}}
$$

with

$$
\begin{aligned}
c_{0} & =\frac{\epsilon_{\lim , 0}\left(s_{\max }\right)}{\eta}, \\
c_{1} & =-2 \frac{A y_{\lim }\left(s_{\max }\right)}{\eta \beta_{y}\left(s_{\max }\right)}, \quad \text { and } \quad c_{2}=\frac{A^{2}}{\eta \beta_{y}\left(s_{\max }\right)} .
\end{aligned}
$$

The coefficients, $c_{0}, c_{1}, c_{2}$, found with the quadratic regression are presented in Table IV. In the regression, the loss times for $y_{\mathrm{co}, 0}=0$ written in the first row of Table III are not used, because in this case it is unlikely that the particles have become lost at the position of the closed orbit bump. Figure 3 shows the resulting parabola compared to the loss times $\tau_{\text {loss }, i}$. The parabola fits the loss times in good approximation. The relative regression error is

TABLE IV. Coefficients found from the quadratic regression.

\begin{tabular}{lr}
\hline \hline$c_{0}$ & $1.023 \times 10^{6}$ \\
$c_{1}\left(\mathrm{~m}^{-1}\right)$ & $-6.9705 \times 10^{7}$ \\
$c_{2}\left(\mathrm{~m}^{-2}\right)$ & $1.1794 \times 10^{9}$ \\
\hline \hline
\end{tabular}

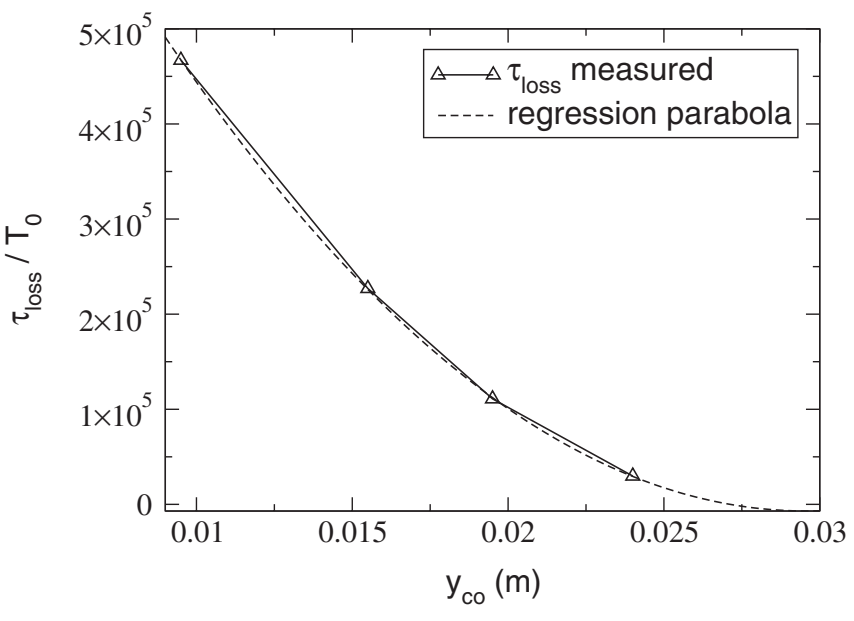

FIG. 3. Loss times determined from the measured current shown in Fig. 2 and the corresponding regression parabola as a function of the closed orbit bump $y_{\mathrm{co}, i}$ for $U_{a}=29 \mathrm{~V}, f_{S}=$ $0.005 / T_{0}$, and $E=100 \mathrm{MeV} / \mathrm{u}$.

$$
\begin{aligned}
\Delta \tau_{\text {loss }, \text { rel }} & =\frac{\sqrt{\sum_{i=1}^{4}\left(\tau_{\text {loss }, i} / T_{0}-c_{0}-c_{1} y_{\mathrm{co}, i}-c_{2} y_{\mathrm{co} . i}^{2}\right)^{2}}}{\sqrt{\sum_{i=1}^{4}\left(\tau_{\text {loss }, i} / T_{0}\right)^{2}}} \\
& \approx 0.005 .
\end{aligned}
$$

This result shows that the condition $\tau_{\text {loss }} \propto \epsilon_{\text {lim }}$ is fulfilled and thus the noise driven emittance growth in SIS-18 using our setup approximates very well the theory presented in Sec. II.

\section{B. Determination of the acceptance}

Finally, we apply the method introduced in Sec. IV to determine the acceptance of SIS-18. The experimental conditions are given by the parameters presented in Table I. The number of tracked particles is $N_{p}=5000$. The number of revolutions is $n \approx 1.2 \times 10^{6}$ corresponding to a time interval of $2 \mathrm{~s}$. We evaluate the experimental data measured for a beam energy $E=100 \mathrm{MeV} / \mathrm{u}$, an exciter amplitude voltage $U_{a}=29 \mathrm{~V}$, and two values of the width of the power spectrum of the exciter, $f_{S}=0.005 / T_{0}$ and $f_{S}=0.01 / T_{0}$.

To find the acceptance we set a test acceptance and simulate the time evolution of the beam current. With a trial-and-error procedure we repeat the simulation with the varied test acceptance until the curve for the particle number fits best the corresponding measured beam current. The test acceptance has been varied between 30 and $80 \mathrm{~mm}$ mrad.

To quantify the difference between measured and calculated particle number, the integral of the square of the difference between both curves for $t>t_{0}$ is determined. The instant $t_{0}$ must be large enough to be in the period of exponential particle loss introduced in Sec. II A. We found this requirement approximately fulfilled for $t_{0}=1.0 \mathrm{~s}$ when $f_{S}=0.01 / T_{0}$ and for $t_{0}=0.75 \mathrm{~s}$ when $f_{S}=0.005 / T_{0}$. The best agreement between measured and calculated 
curves of the current is found for an acceptance $\epsilon_{\text {lim }}=$ $46 \mathrm{~mm} \mathrm{mrad}$ for $f_{S}=0.01 / T_{0}$ and $\epsilon_{\mathrm{lim}}=45 \mathrm{~mm} \mathrm{mrad}$ for $f_{S}=0.005 / T_{0}$. Performing an error analysis, we have estimated the maximum error due to uncertainties in the lattice and beam parameters to be smaller than $13 \%$. The details of this analysis are presented in Appendix B.

\section{SUMMARY}

We applied a vertical rf voltage to determine the vertical acceptance of the GSI heavy ion synchrotron, SIS-18. The excitation of the beam by the rf field led to an increase of the vertical beam size and to a reduction of the beam current when particles reached the aperture. We measured the beam current as a function of time and extracted the acceptance from the loss rate. The aim of this method was to remove the dependence of the measured data on the initial beam width.

The experimental data were quantitatively evaluated via particle tracking. First, we benchmarked the tracking method against an analytic model, where we used white noise in our numerical code and obtained very good agreement. To analyze the experimental data we applied the rf excitation used in the current measurements.

Finally, we determined the acceptance of SIS-18 using a trial-and-error procedure, where we repeated the simulations with many different test acceptances until we found one so that the resulting time evolution of the particle number fits the measured beam current.

In addition, we performed a numerical study where we varied beam and lattice parameters in order to estimate an upper limit for the acceptance error. We found that some of the parameter errors caused an acceptance error because the power spectrum of the exciter had a width $f_{S}$ similar to the spread of the betatron frequency of the particles. For this reason, there is a betatron frequency dependence in the amount of excitation each particle receives because of the nonuniform power density. Consequently, we found that the modification of the acceptance due to the variation of these parameters depends on the width of the noise power spectrum. However, we did find nearly the same acceptance for different values of $f_{S}$. This suggests that the size of $f_{S}$ does not dominate the acceptance error. We conclude that our method can reproduce the acceptance of an accelerator with an acceptable accuracy.

\section{ACKNOWLEDGMENTS}

We thank our colleagues O. Boine-Frankenheim and M. Kirk for helpful hints and discussions, and P. Kowina, W. Kaufmann, and C. Wetzel for help in the preparation and the usage of the exciter.

\section{APPENDIX A: TIME EVOLUTION OF THE AVERAGED EMITTANCE DUE TO WHITE NOISE}

In this Appendix, the time evolution of the beam emittance, $\epsilon_{\mathrm{av}}$, is calculated in terms of the number of revolutions, $n=t / T_{0}$, where each particle experiences in every revolution a random momentum kick. As discussed in Sec. II B, these kicks are assumed to be uncorrelated with respect to the particle and the revolution. The starting point is Eq. (17),

$$
\begin{aligned}
\epsilon_{\mathrm{av}}(n)= & \frac{1}{N_{p}} \sum_{p=1}^{N_{p}}\left\{\left(y_{p, 0}, y_{p, 0}^{\prime}\right) \cdot\left(M^{n}\right)^{T} \cdot B \cdot M^{n} \cdot\left(\begin{array}{c}
y_{p, 0} \\
y_{p, 0}^{\prime}
\end{array}\right)\right. \\
& +\sum_{k=0}^{n-1}\left[\left(y_{p, 0}, y_{p, 0}^{\prime}\right) \cdot\left(M^{n}\right)^{T} \cdot B \cdot M^{n-k} \cdot\left(\begin{array}{c}
0 \\
\Delta y_{p, k}^{\prime}
\end{array}\right)\right. \\
& \left.+\left(0, \Delta y_{p, k}^{\prime}\right) \cdot\left(M^{n-k}\right)^{T} \cdot B \cdot M^{n}\left(\begin{array}{c}
y_{p, 0} \\
y_{p, 0}^{\prime}
\end{array}\right)\right] \\
& \left.+\sum_{k, l=0}^{n-1}\left(0, \Delta y_{p, l}^{\prime}\right) \cdot\left(M^{n-l}\right)^{T} \cdot B \cdot M^{n-k} \cdot\left(\begin{array}{c}
0 \\
\Delta y_{p, k}^{\prime}
\end{array}\right)\right\},
\end{aligned}
$$

where

$$
\left(\begin{array}{c}
y_{p, n} \\
y_{p, n}^{\prime}
\end{array}\right)=M^{n}\left(\begin{array}{l}
y_{0} \\
y_{0}^{\prime}
\end{array}\right)+\sum_{k=0}^{n-1} M^{n-k}\left(\begin{array}{c}
0 \\
\Delta y_{p, k}^{\prime}
\end{array}\right)
$$

is the phase space vector of the $p$ th particle after $n$ revolutions according to Eq. (16). The beta matrix $B$ containing the Twiss functions reads

$$
B=\left(\begin{array}{cc}
\frac{1+\alpha^{2}}{\beta} & \alpha \\
\alpha & \beta
\end{array}\right)
$$

In Eq. (A1), the matrix

$$
M=\left(\begin{array}{cc}
\cos \mu+\alpha \sin \mu & \beta \sin \mu \\
-\frac{1+\alpha^{2}}{\beta} \sin \mu & \cos \mu-\alpha \sin \mu
\end{array}\right)
$$

is a linear one turn map providing the time evolution of one particle during a single revolution.

The transformation of the phase space coordinates of a particle due to $M$ does not change its emittance. Hence, the first term in the curly brackets in Eq. (A1) gives the initial beam emittance $\epsilon_{\mathrm{av}, 0}$, and the change of the beam emittance during $n$ revolutions,

$$
\Delta \epsilon_{\mathrm{av}}(n)=\epsilon_{\mathrm{av}}(n)-\epsilon_{\mathrm{av}, 0},
$$

arises from the sum over the second and the third term in the curly brackets in Eq. (A1).

The contribution arising from the terms in the square brackets in Eq. (A1) vanishes because, according to Eqs. (19) and (20),

$$
\frac{1}{N_{p}} \sum_{p=1}^{N_{p}} \Delta y_{p, k}^{\prime}=0
$$

and the kicks $\Delta y_{p, k}^{\prime}$ do not depend on the initial phase space coordinates of the particles $\left(y_{p, 0}, y_{p, 0}^{\prime}\right)$. 
To perform the ensemble average of the last term in the curly brackets of Eq. (A1), the correlation function with respect to the revolution number,

$$
\frac{1}{N_{p}} \sum_{p=1}^{N_{p}} \Delta y_{p, l}^{\prime} \Delta y_{p, k}^{\prime}=\sigma_{\Delta y^{\prime}}^{2} \delta_{l k},
$$

can be used, which approaches the mean squared value of the single-kick distribution $p_{1}\left(\Delta y^{\prime}\right)$ given in Eq. (21) when $l=k$ and $N_{p} \rightarrow \infty$. Therefore, we find that the change in beam emittance defined in Eq. (A5) is

$$
\begin{aligned}
\Delta \epsilon_{\mathrm{av}}(n) & =\frac{1}{N_{p}} \sum_{p=1}^{N_{p}} \sum_{k=0}^{n-1}\left(0, \Delta y_{p, k}^{\prime}\right) \cdot\left(M^{n-k}\right)^{T} \cdot B \cdot M^{n-k} \cdot\left(\begin{array}{c}
0 \\
\Delta y_{p, k}^{\prime}
\end{array}\right) \\
& =\sigma_{\Delta y^{\prime}}^{2} \sum_{k=0}^{n-1}(0,1) \cdot\left(M^{n-k}\right)^{T} \cdot B \cdot M^{n-k} \cdot\left(\begin{array}{l}
0 \\
1
\end{array}\right) \\
& =n \beta \sigma_{\Delta y^{\prime}}^{2} .
\end{aligned}
$$

\section{APPENDIX B: ERROR ESTIMATION}

The lattice and beam parameters used in the calculations are standard values used in the machine control system, or they are based on a calculation done with MAD-X [11]. That implies there may be deviations of these parameters from those in the real machine.

In this analysis, we have to distinguish between random errors which can be different for different measurements under the same conditions and systematic errors which remain constant as long as the machine settings are not modified.

In the first step we investigate the random errors. Random errors lead to a spread of the measured curves of the current $I(t)$. Therefore, we estimate the random errors by direct comparison of all curves of $I(t)$ measured for the same parameters. These curves have been adjusted so that they pass through the same value at $t_{0}=1.0 \mathrm{~s}$ for $f_{S}=0.01 / T_{0}$ and $t_{0}=0.75 \mathrm{~s}$ for $f_{S}=0.005 / T_{0}$, where $t_{0}$ denotes approximately the beginning of the period of exponentially decreasing particle number. The maximum spread in $I(t)$ has always been found to be smaller than $1.0 \%$ which corresponds to an acceptance error up to $\left|\Delta \epsilon_{\text {lim,random }}\right|=1 \mathrm{~mm} \operatorname{mrad}$ or $\delta \epsilon_{\text {lim,random }} \equiv$ $\left|\Delta \epsilon_{\text {lim,random }}\right| / \epsilon_{\text {lim }}=0.02$.

To estimate the effect of systematic errors, we study numerically the dependence of the acceptance on the uncertainties of the parameters $X \equiv\left(\beta_{y}, \alpha_{y}, \sigma_{p}, \xi_{y}\right)$. In the study we add a maximum error $\Delta X$ to each parameter $X_{0}$ from Table I which we use to determine the acceptance, where only one of the parameters is changed for each simulation. Applying the trial-and-error procedure introduced in Sec. V B, we find the acceptance $\epsilon_{\lim }\left(X_{0}+\Delta X\right)$ so that the resulting particle number $N(t)$ fits the best $N(t)$ calculated from $X_{0}$. The difference between $\epsilon_{\lim }\left(X_{0}+\Delta X\right)$
TABLE V. Variables and their assumed maximum systematic relative deviations.

\begin{tabular}{lcc}
\hline \hline Variable & Unperturbed value & Maximum relative error \\
\hline & (see Table I) & $\left|\Delta X / X_{0}\right|_{\max }$ \\
$\beta_{y}$ & $7.0 \mathrm{~m}$ & $10 \%$ \\
$\xi_{y}$ & -1.4647 & $10 \%$ \\
$\sigma_{p}$ & $5 \times 10^{-4}$ & $10 \%$ \\
\hline \hline
\end{tabular}

and $\epsilon_{\text {lim }}\left(X_{0}\right)$ is the systematic error arising from $\Delta X$. The maximum relative errors $\left|\Delta X / X_{0}\right|_{\max }$ are shown in Table $\mathrm{V}$. We assume these errors to describe the worst case in order to find an upper limit for the acceptance error.
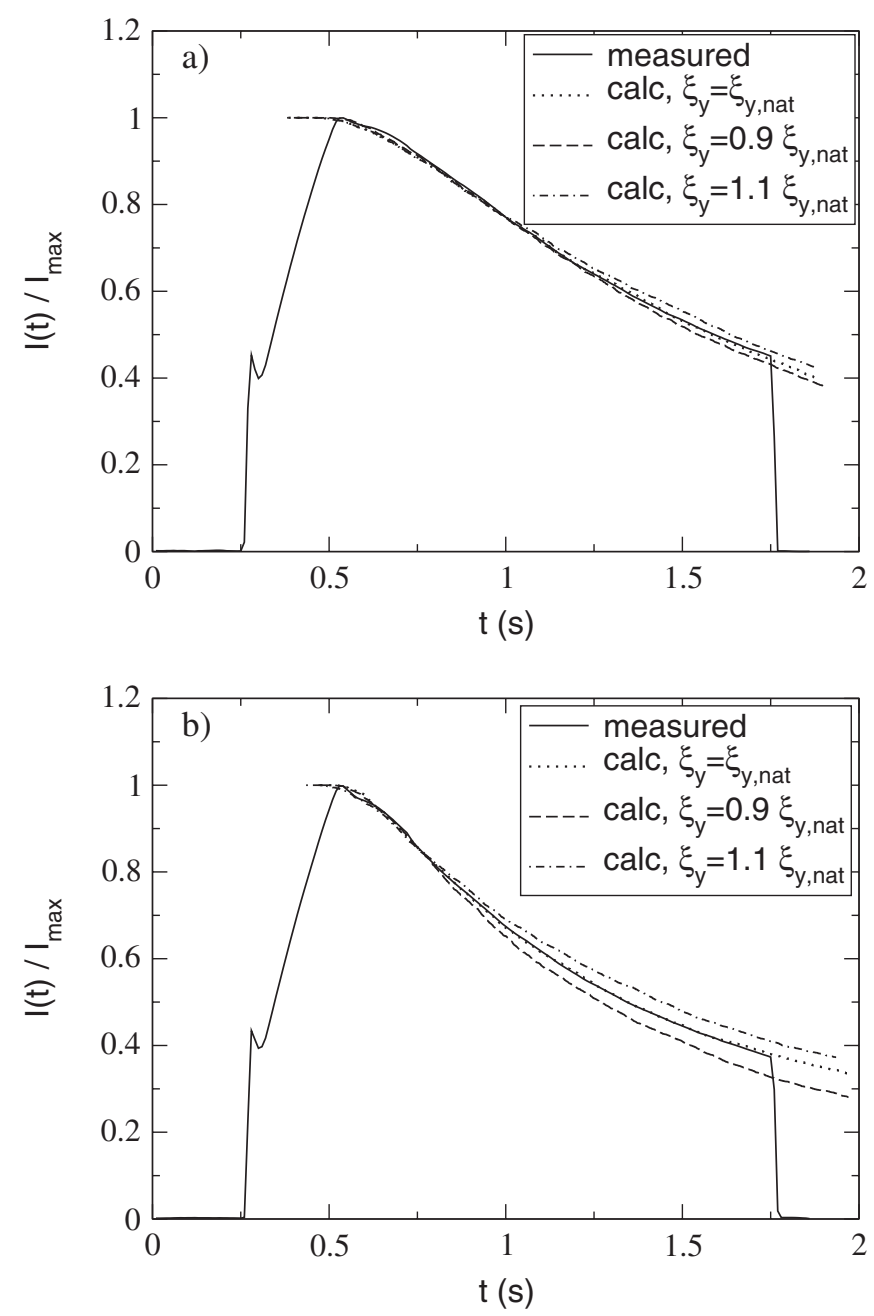

FIG. 4. Current as a function of time measured and computed for $U_{a}=29 \mathrm{~V}$. In graph (a), the half width of the power spectrum of the exciter [Eq. (26)] is $f_{S}=0.01 / T_{0}$ and the acceptance is $\epsilon_{\mathrm{lim}}=46 \mathrm{~mm}$ mrad. In graph (b), $f_{S}=0.005 / T_{0}$ and $\epsilon_{\text {lim }}=45 \mathrm{~mm}$ mrad are used. For both graphs the chromaticity has been varied by $\pm 10 \%$ in the simulations. The resulting modifications of the beam current $I(t)$ at $t=1.75 \mathrm{~s}$ are $+4.5 \%$ and $-2.7 \%$, respectively, in graph (a) and $+7.2 \%$ and $-14.5 \%$, respectively, in graph (b). 
The results obtained from the variation of $\alpha_{y}$ and $\beta_{y}$ can be checked using a simplification of Eq. (17). Replacing the random momentum kick by that of the exciter given in Eq. (28) and the one turn map $M(\mu)$ by $M\left(\mu_{p}\right)$ according to Eq. (33), we find that the time evolution of the beam emittance is

$$
\epsilon_{\mathrm{av}}(n)=\epsilon_{\mathrm{av}, 0}+\frac{\beta_{y}}{N_{p}} \sum_{p=1}^{N_{p}} \sum_{k, l=1}^{n-1} \cos \left[(k-l) \mu_{p}\right] \Delta y_{k}^{\prime} \Delta y_{l}^{\prime},
$$

where $\mu_{p}=\mu+\mu \xi_{y} \delta_{p}$ is the phase advance of the $p$ th particle per revolution. This equation shows that $\epsilon_{\mathrm{av}}$ is independent of $\alpha_{y}$ and it is only a function of $\beta_{y}$. Therefore, there is no error for $\alpha_{y}$ in Table V. The predictions of Eq. (B1) are numerically confirmed to a high precision, i.e., the acceptance error due to the error in the beta function of $10 \%$ is

$$
\delta \epsilon_{\lim }\left(\Delta \beta_{y}\right) \equiv\left|\Delta \epsilon_{\lim }\left(\Delta \beta_{y}\right) / \epsilon_{\lim }\right|=0.1 \text {. }
$$

The uncertainties of $\xi_{y}$ and $\sigma_{p}$ yield the uncertainty of the rms betatron tune of the particles $\Delta \nu_{\mathrm{rms}}=\Delta\left(\nu_{y} \xi_{y} \sigma_{p}\right)$ that influences the growth of the beam emittance because the corresponding spread in the particle betatron frequencies is similar to the width of the noise power spectrum of the exciter $f_{S}$. That leads to a nonuniform spectral power density so that the excitation of a particle depends on its tune. Applying an error in $\xi_{y}$ of $\pm 10 \%$, we found that the resulting modification of the beam current $I(t)$ is strongly influenced by $f_{S}$, which one can see in Fig. 4. Consequently, the acceptance error is strongly influenced by $f_{S}$ because the acceptance needs to be changed by -2.5 and $+3.7 \mathrm{mmmrad}$, respectively, so that $N(t)$ fits the calculated curve when $f_{S}=0.01 / T_{0}$, whereas for $f_{S}=0.005 / T_{0}$ the change is $\mp 6.6 \mathrm{~mm} \mathrm{mrad}$. However, $\epsilon_{\lim }$ must not depend on $f_{S}$ and so the initial estimate for the error in $\xi_{y}$ is too large. By reducing the chromaticity error to $-4 \%, \epsilon_{\lim }$ becomes independent of $f_{S}$. Using this new error estimate for $\xi_{y}$, we find that $\epsilon_{\text {lim }}=48 \mathrm{~mm} \mathrm{mrad}$. The relative deviation of this value from the smaller acceptance calculated for unperturbed parameters, $\epsilon_{\lim }=$ $45 \mathrm{~mm} \mathrm{mrad}$, is

$$
\delta \epsilon_{\lim }\left[\Delta\left(\xi_{y} \sigma_{p}\right)\right] \equiv\left|\Delta \epsilon_{\lim }\left[\Delta\left(\xi_{y} \sigma_{p}\right)\right] / \epsilon_{\lim }\right|=0.067
$$

If the uncertainties in $\beta_{y}$ and $\xi_{y} \sigma_{p}$ are independent then the resulting total acceptance error is $\left|\Delta \epsilon_{\lim } / \epsilon_{\text {lim }}\right|_{\text {tot }}=$ $\sqrt{\left[\delta \epsilon_{\text {lim,random }}\right]^{2}+\left[\delta \epsilon_{\text {lim }}\left(\Delta \beta_{y}\right)\right]^{2}+\left\{\delta \epsilon_{\lim }\left[\Delta\left(\xi_{y} \sigma_{p}\right)\right]\right\}^{2}}<$ 0.13 . According to the values for the acceptance in the previous section, $\epsilon_{\mathrm{lim}}$ is between 40 and $52 \mathrm{~mm}$ mrad.

[1] Parameter list of SIS-18: http://www.gsi.de/beschleuniger/ sis18/pdf/sis18_parameterliste.pdf.

[2] P. Spiller and G. Franchetti, Nucl. Instrum. Methods Phys. Res., Sect. A 561, 305 (2006).

[3] "An International Accelerator Facility for Beams of Ions and Antiprotons," Conceptual design report, GSI Darmstadt, 2006, Section 3, "The Facility," http:// www.gsi.de/GSI-Future/cdr.

[4] E. Mustafin, O. Boine-Frankenheim, I. Hofmann, H. Reich-Sprenger, and P. Spiller, Nucl. Instrum. Methods Phys. Res., Sect. A 510, 199 (2003).

[5] C. Omet, P. Spiller, J. Stadlmann, and D. H. H. Hoffmann, New J. Phys. 8, 284 (2006).

[6] A. Parfenova and G. Franchetti, in Proceedings of the 23rd Particle Accelerator Conference, Vancouver, Canada, 2009 (IEEE, Piscataway, NJ, 2009), TH6PFP053, p. 3826.

[7] X. L. Zhang, V. Shiltsev, and C. Y. Tan, in Proceedings of the 21st Particle Accelerator Conference, Knoxville, 2005 (IEEE, Piscataway, NJ, 2005), p. 2306.

[8] X. Altuna, A. Arimatea, R. Bailey, T. Bohl, D. Brandt, K. Cornelis, C. Depas, F. Gallucio, J. Gareyte, R. Giachino, M Giovannozzi, Z. Guo, W. Herr, A. Hilaire, T. Lundberg, J. Miles, L. Normann, T. Risselada, W. Scandale, F. Schmidt, A. Spinks, and M. Venturini, The 1991 Dynamic Aperture Experiment at the CERN SPS, in AIP Conf. Proc. No. 255 (AIP, New York, 1991), p. 355.

[9] D. A. Edwards and M. Syphers, An Introduction to the Physics of High Energy Accelerators (Wiley, New York, 1993).

[10] M. Kirk and P. Moritz, GSI Internal Note, GSI Darmstadt, 2006.

[11] MAD-X code, see http://mad.web.cern.ch/mad. 